\title{
The Kirchhoff Index of Toroidal Meshes and Variant Networks
}

\author{
Jia-Bao Liu, ${ }^{1,2,3}$ Xiang-Feng Pan, ${ }^{1}$ Jinde Cao, ${ }^{2,4}$ and Xia Huang ${ }^{5}$ \\ ${ }^{1}$ School of Mathematical Sciences, Anhui University, Hefei 230601, China \\ ${ }^{2}$ Department of Mathematics, Southeast University, Nanjing 210096, China \\ ${ }^{3}$ Department of Public Courses, Anhui Xinhua University, Hefei 230088, China \\ ${ }^{4}$ Department of Mathematics, Faculty of Science, King Abdulaziz University, Jeddah 21589, Saudi Arabia \\ ${ }^{5}$ College of Electrical Engineering and Automation, Shandong University of Science and Technology, Qingdao 266590, China
}

Correspondence should be addressed to Jinde Cao; jdcao@seu.edu.cn

Received 14 March 2014; Accepted 20 May 2014; Published 3 June 2014

Academic Editor: He Huang

Copyright (C) 2014 Jia-Bao Liu et al. This is an open access article distributed under the Creative Commons Attribution License, which permits unrestricted use, distribution, and reproduction in any medium, provided the original work is properly cited.

\begin{abstract}
The resistance distance is a novel distance function on electrical network theory proposed by Klein and Randić. The Kirchhoff index $\mathrm{Kf}(G)$ is the sum of resistance distances between all pairs of vertices in $G$. In this paper, we established the relationships between the toroidal meshes network $T_{m \times n}$ and its variant networks in terms of the Kirchhoff index via spectral graph theory. Moreover, the explicit formulae for the Kirchhoff indexes of $L\left(T_{m \times n}\right), S\left(T_{m \times n}\right), T\left(T_{m \times n}\right)$, and $C\left(T_{m \times n}\right)$ were proposed, respectively. Finally, the asymptotic behavior of Kirchhoff indexes in those networks is obtained by utilizing the applications of analysis approach.
\end{abstract}

\section{Introduction}

Throughout this paper we are concerned with finite undirected connected simple graphs (networks). Let $G=(V, E)$ be a graph with vertices labelled $1,2, \ldots, n$. The adjacency matrix $A(G)$ of $G$ is an $n \times n$ matrix with the $(i, j)$-entry equal to 1 if vertices $i$ and $j$ are adjacent and 0 otherwise. Suppose $D(G)=\operatorname{diag}\left(d_{1}(G), d_{2}(G), \ldots, d_{n}(G)\right)$ is the degree diagonal matrix of $G$, where $d_{i}(G)$ is the degree of the vertex $i$, $i=1,2, \ldots, n$. Let $L(G)=D(G)-A(G)$ be called the Laplacian matrix of $G$. Then, the eigenvalues of $A(G)$ and $L(G)$ are called eigenvalues and Laplacian eigenvalues of $G$, respectively.

Given graphs $G$ and $H$ with vertex sets $U$ and $V$, the Cartesian product $G \square H$ of graphs $G$ and $H$ is a graph such that the vertex set of $G \square H$ is the Cartesian product $U \times V$; and any two vertices $\left(u, u^{\prime}\right)$ and $\left(v, v^{\prime}\right)$ are adjacent in $G \square H$ if and only if either $u=v$ and $u^{\prime}$ is adjacent with $v^{\prime}$ in $H$ or $u^{\prime}=v^{\prime}$ and $u$ is adjacent with $v$ in G [1]. It is well known that many of the graphs (networks) operations can produce a great deal of novel types of graphs (networks), for example, Cartesian product of graphs, line graph, subdivision graph, and so on. The clique-inserted graph, denoted by $C(G)$, is defined as a line graph of the subdivision graph $S(G)$
$[2,3]$. The subdivision graph of an $r$-regular graph is $(r, 2)$ semiregular graph. Consequently, the clique-inserted graph of an $r$-regular graph is the line graph of an $(r, 2)$-semiregular graph.

The resistance distances between vertices $i$ and $j$, denoted by $r_{i j}$, are defined as the effective electrical resistance between them if each edge of $G$ is replaced by a unit resistor [4]. A famous distance-based topological index, the Kirchhoff index $\operatorname{Kf}(G)$, is defined as the sum of resistance distances between all pairs of vertices in $G$; that is, $\operatorname{Kf}(G)=$ $(1 / 2) \sum_{i=1}^{n} \sum_{j=1}^{n} r_{i j}(G)$, known as the Kirchhoff index of $G[4]$; recently, this classical index has also been interpreted as a measure of vulnerability of complex networks [5].

The Kirchhoff index attracted extensive attention due to its wide applications in physics, chemistry, graph theory, and so forth [6-13]. Besides, the Kirchhoff index also is a structure descriptor [14]. Unfortunately, it is rather hard to directly design some algorithms [15-17] to calculate resistance distances and the Kirchhoff indexes of graphs. So, many researchers investigated some special classes of graphs [1821]. In addition, many efforts were also made to obtain the Kirchhoff index bounds for some graphs [17, 22]. Details on 
its theory can be found in recent papers [17, 22] and the references cited therein.

Motivated by the above results, we present the corresponding calculating formulae for the Kirchhoff index of $L\left(T_{m \times n}\right), S\left(T_{m \times n}\right), T\left(T_{m \times n}\right)$, and $C\left(T_{m \times n}\right)$ in this paper. The rest of this paper is organized as follows. Section 2 presents some underlying notations and preliminaries in our discussion. The proofs of our main results and some asymptotic behavior of Kirchhoff index are proposed in Sections 3 and 4 , respectively.

\section{Notations and Some Preliminaries}

In this section, we introduced some basic properties which we need to use in the proofs of our main results. Suppose that $T_{m \times n}$ stands for the graphs $C_{m} \square C_{n}$ for the convenience of description. It is trivial for $m, n$ are 1,2 , without loss of generality, we discuss the situations for any positive integer $m, n \geq 3$.

Zhu et al. [15] and Gutman and Mohar [8] proved the relations between Kirchhoff index of a graph and Laplacian eigenvalues of the graph as follows.

Lemma 1 (see $[8,15])$. Let $G$ be a connected graph with $n \geq 2$ vertices and let $\mu_{1} \geq \mu_{2} \geq \cdots \geq \mu_{n}=0$ be the Laplacian eigenvalues of graph $G$; then

$$
K f(G)=n \sum_{i=1}^{n-1} \frac{1}{\mu_{i}}
$$

The line graph of a graph $G$, denoted by $L(G)$, is the graph whose vertices correspond to the edges of $G$ with two vertices of $L(G)$ being adjacent if and only if the corresponding edges in $G$ share a common vertex. The subdivision graph of a graph $G$, denoted by $S(G)$, is the graph obtained by replacing every edge in $G$ with a copy of $P_{2}$ (path of length two). The total graph of a graph $G$, denoted by $T(G)$, is the graph whose vertices correspond to the union of the set of vertices and edges of $G$, with two vertices of $T(G)$ being adjacent if and only if the corresponding elements are adjacent or incident in $G$. Let $P_{G}(x)$ be the characteristic polynomial of the Laplacian matrix of a graph $G$; the following results were shown in [23].

Lemma 2 (see [23]). Let $G$ be an r-regular connected graph with $n$ vertices and $m$ edges; then

$$
\begin{gathered}
P_{L(G)}(x)=(x-2 r)^{m-n} P_{G}(x), \\
P_{S(G)}(x)=(-1)^{m}(2-x)^{m-n} P_{G}(x(r+2-x)), \\
P_{T(G)}(x) \\
=(-1)^{m}(r+1-x)^{n}(2 r+2-x)^{m-n} P_{G}\left(\frac{x(r+2-x)}{r-x+1}\right),
\end{gathered}
$$

where $P_{L(G)}(x), P_{S(G)}(x)$, and $P_{T(G)}(x)$ are the characteristic polynomials for the Laplacian matrix of graphs $L(G), S(G)$, and $T(G)$, respectively.
A bipartite graph $G$ with a bipartition $V(G)=(U, V)$ is called an $(r, s)$-semiregular graph if all vertices in $U$ have degree $r$ and all vertices in $V$ have degree $s$. Apparently, the subdivision graph of an $r$-regular-graph $G$ is $(r, 2)$ semiregular graph.

Lemma 3 (see [24]). Let $G$ be an $(r, s)$-semiregular connected graph with $n$ vertices. Then

$$
P_{L(G)}(x)=(-1)^{n}(x-(r+s))^{m-n} P_{G}(r+s-x),
$$

where $P_{L(G)}(x)$ is the Laplacian characteristic polynomial of the line graph $L(G)$ and $m$ is the number of edges of $G$.

Lemma 4 (see [23]). Let $G$ be a connected simple r-regular graph with $n$ vertices and $m$ edges and let $L(G)$ be the line graph of $G$. Then

$$
K f(L(G))=\frac{r}{2} K f(G)+\frac{1}{4} n(m-n) .
$$

Lemma 5 (see [23]). Let $G$ be a connected simple r-regular graph with $n \geq 2$ vertices; then

$$
K f(S(G))=\frac{(r+2)^{2}}{2} K f(G)+\frac{\left(r^{2}-4\right) n^{2}+4 n}{8} .
$$

The following lemma gives an expression on $\operatorname{Kf}(T(G))$ and $\mathrm{Kf}(G)$ of a regular graph $G$.

Lemma 6 (see [25]). Let $G$ be a r-regular connected graph with $n$ vertices and $m$ edges, and $r \geq 2$; then

$$
\begin{aligned}
K f(T(G))= & \frac{n(r+2)(r+4)}{2(r+3)} \sum_{i=1}^{n-1} \frac{1}{\mu_{i}+3+r} \\
& +\frac{(r+2)^{2}}{2(r+3)} K f(G)+\frac{n^{2}\left(r^{2}-4\right)}{8(r+1)}+\frac{n}{2} .
\end{aligned}
$$

Lemma 7 presents the formula for calculating Kirchhoff index of $T_{m \times n}$; in the following proof, some techniques in [26] are referred to.

Lemma 7 (see [26]). For the toroidal networks $T_{m \times n}$ with any positive integer $m, n \geq 3$,

$$
\begin{aligned}
K f\left(T_{m \times n}\right)= & m n \sum_{i=1}^{m-1} \sum_{j=1}^{n-1} \frac{1}{4 \sin ^{2}(i \pi / m)+4 \sin ^{2}(j \pi / n)} \\
& +n \frac{m^{3}-m}{12}+m \frac{n^{3}-n}{12} .
\end{aligned}
$$

Proof. Suppose the Laplacian eigenvalues of $C_{m}$ and $C_{n}$ are $4 \sin ^{2}(i \pi / m)$ and $4 \sin ^{2}(j \pi / n), i=0,1, \ldots, m-1 ; j=$ $0,1, \ldots, n-1$; then the Cartesian product $G \square H$ and the Laplacian eigenvalues of $L_{\left(c_{m} \square C_{n}\right)}$ are

$$
4 \sin ^{2} \frac{i \pi}{m}+4 \sin ^{2} \frac{j \pi}{n}, \quad i=0,1, \ldots, m-1 ; j=0,1, \ldots, n-1 .
$$


According to Lemma 1, the Kirchhoff index of the toroidal networks $\mathrm{Kf}\left(T_{m \times n}\right)$ is

$$
\begin{aligned}
& \operatorname{Kf}\left(T_{m \times n}\right)=m n \sum_{(i, j) \in A \times B \backslash\{(0,0)\}} \frac{1}{4 \sin ^{2}(i \pi / m)+4 \sin ^{2}(j \pi / n)}, \\
& A=\{0,1, \ldots, m-1\}, \quad B=\{0,1, \ldots, n-1\} \\
& =m n \sum_{i=0}^{m-1} \sum_{j=0}^{n-1} \frac{1}{4 \sin ^{2}(i \pi / m)+4 \sin ^{2}(j \pi / n)}, \\
& (i, j) \neq(0,0) \\
& =m n\left(\sum_{i=1}^{m-1} \frac{1}{4 \sin ^{2}(i \pi / m)}+\sum_{j=1}^{n-1} \frac{1}{4 \sin ^{2}(j \pi / n)}\right. \\
& \left.+\sum_{i=1}^{m-1} \sum_{j=1}^{n-1} \frac{1}{4 \sin ^{2}(i \pi / m)+4 \sin ^{2}(j \pi / n)}\right) \\
& =n\left(m \sum_{i=1}^{m-1} \frac{1}{4 \sin ^{2}(i \pi / m)}\right) \\
& +m\left(n \sum_{j=1}^{n-1} \frac{1}{4 \sin ^{2}(j \pi / n)}\right) \\
& +m n \sum_{i=1}^{m-1} \sum_{j=1}^{n-1} \frac{1}{4 \sin ^{2}(i \pi / m)+4 \sin ^{2}(j \pi / n)} \\
& =n \operatorname{Kf}\left(C_{m}\right)+m \operatorname{Kf}\left(C_{n}\right) \\
& +m n \sum_{i=1}^{m-1} \sum_{j=1}^{n-1} \frac{1}{4 \sin ^{2}(i \pi / m)+4 \sin ^{2}(j \pi / n)} \\
& =m n \sum_{i=1}^{m-1} \sum_{j=1}^{n-1} \frac{1}{4 \sin ^{2}(i \pi / m)+4 \sin ^{2}(j \pi / n)} \\
& +n \frac{m^{3}-m}{12}+m \frac{n^{3}-n}{12} \text {. }
\end{aligned}
$$

\section{Main Results}

3.1. The Kirchhoff Index of $L\left(T_{m \times n}\right)$. In the following theorem, we proposed the formula for calculating the Kirchhoff index of the line graph of $T_{m \times n}$, denoted by $\operatorname{Kf}\left(L\left(T_{m \times n}\right)\right)$.

Theorem 9. Let $L\left(T_{m \times n}\right)$ be line graphs of $T_{m \times n}$ with any positive integer $m, n \geq 3$; then

$$
\begin{aligned}
K f\left(L\left(T_{m \times n}\right)\right)= & 2 m n \sum_{i=1}^{m-1} \sum_{j=1}^{n-1} \frac{1}{4 \sin ^{2}(i \pi / m)+4 \sin ^{2}(j \pi / n)} \\
& +n \frac{m^{3}-m}{6}+m \frac{n^{3}-n}{6}+\frac{m^{2} n^{2}}{4} .
\end{aligned}
$$

Proof. Apparently the toroidal networks $T_{m \times n}$ are 4-regular graphs which have $m n$ vertices and $2 m n$ edges, respectively.

We clearly obtained the following relationship $\mathrm{Kf}\left(L\left(T_{m \times n}\right)\right)$ and $\mathrm{Kf}\left(T_{m \times n}\right)$ from Lemma 4:

$$
\begin{aligned}
\mathrm{Kf}\left(L\left(T_{m \times n}\right)\right) & =\frac{r}{2} \operatorname{Kf}\left(T_{m \times n}\right)+\frac{(r-2) m^{2} n^{2}}{8} \\
& =2 \mathrm{Kf}\left(T_{m \times n}\right)+\frac{m^{2} n^{2}}{4} .
\end{aligned}
$$

Since $\operatorname{Kf}\left(C_{n}\right)=\left(n^{3}-n\right) / 12,(10)$ in the last line holds.

The following consequence was presented in [26]. Here we give a short proof.

Lemma 8 (see [26]). For the toroidal networks $T_{m \times n}$ with any positive integer $m, n \geq 3$,

$$
\lim _{m \rightarrow \infty} \lim _{n \rightarrow \infty} \frac{K f\left(T_{m \times n}\right)}{m^{2} n^{2}} \approx 1.905 .
$$

Proof. By virtue of (9), one can derive that

$$
\begin{array}{r}
\frac{\mathrm{Kf}\left(T_{m \times n}\right)}{m n}=\sum_{i=0}^{m-1} \sum_{j=0}^{n-1} \frac{1}{4 \sin ^{2}(i \pi / m)+4 \sin ^{2}(j \pi / n)}, \\
(i, j) \neq(0,0) .
\end{array}
$$

Substituting the results of Lemma 7 into (15), we can get the formula for the Kirchhoff index of $\operatorname{Kf}\left(L\left(T_{m \times n}\right)\right)$,

$$
\begin{aligned}
\operatorname{Kf}\left(L\left(T_{m \times n}\right)\right)= & 2 m n \sum_{i=1}^{m-1} \sum_{j=1}^{n-1} \frac{1}{4 \sin ^{2}(i \pi / m)+4 \sin ^{2}(j \pi / n)} \\
& +n \frac{m^{3}-m}{6}+m \frac{n^{3}-n}{6}+\frac{m^{2} n^{2}}{4},
\end{aligned}
$$

3.2. The Kirchhoff Index of $S\left(T_{m \times n}\right)$. In an almost identical way as Theorem 9, we derived the formula for the Kirchhoff index on the subdivision graph of $T_{m \times n}$, denoted by $\operatorname{Kf}\left(S\left(T_{m \times n}\right)\right)$. which completes the proof. 
Theorem 10. Let $S\left(T_{m \times n}\right)$ be subdivision graphs of $T_{m \times n}$ with any positive integer $m, n \geq 3$; then

$$
\begin{aligned}
K f\left(S\left(T_{m \times n}\right)\right)= & 18 m n \sum_{i=1}^{m-1} \sum_{j=1}^{n-1} \frac{1}{4 \sin ^{2}(i \pi / m)+4 \sin ^{2}(j \pi / n)} \\
& +3 n \frac{m^{3}-m}{2}+3 m \frac{n^{3}-n}{2}+\frac{3 m^{2} n^{2}+m n}{2} .
\end{aligned}
$$

Proof. Noting that $T_{m \times n}$ are 4-regular graphs which have $m n$ vertices, we clearly obtained from Lemma 5

$$
\begin{aligned}
\operatorname{Kf}\left(S\left(T_{m \times n}\right)\right) & =\frac{(r+2)^{2}}{2} \operatorname{Kf}\left(T_{m \times n}\right)+\frac{\left(r^{2}-4\right) m^{2} n^{2}+4 m n}{8} \\
& =18 \mathrm{Kf}\left(T_{m \times n}\right)+\frac{3 m^{2} n^{2}+m n}{2} .
\end{aligned}
$$

Together with the results of Lemma 7 and (18), we can get the formula for the Kirchhoff index on the subdivision graph of $T_{m \times n}$ :

$$
\begin{aligned}
\operatorname{Kf}\left(S\left(T_{m \times n}\right)\right)= & 18 m n \sum_{i=1}^{m-1} \sum_{j=1}^{n-1} \frac{1}{4 \sin ^{2}(i \pi / m)+4 \sin ^{2}(j \pi / n)} \\
& +3 n \frac{m^{3}-m}{2}+3 m \frac{n^{3}-n}{2}+\frac{3 m^{2} n^{2}+m n}{2} .
\end{aligned}
$$

The proof is completed.

3.3. The Kirchhoff Index of $T\left(T_{m \times n}\right)$. Now we proved the formula for estimating the Kirchhoff index in the total graph of $T_{m \times n}$, denoted by $\operatorname{Kf}\left(T\left(T_{m \times n}\right)\right)$.

Theorem 11. Let $T\left(T_{m \times n}\right)$ be total graphs of $T_{m \times n}$ with any positive integer $m, n \geq 3$; then

$$
\begin{aligned}
K f & \left(T\left(T_{m \times n}\right)\right) \\
= & \frac{18}{7} m n \sum_{i=1}^{m-1} \sum_{j=1}^{n-1} \frac{1}{4 \sin ^{2}(i \pi / m)+4 \sin ^{2}(j \pi / n)} \\
& +\frac{24 m n}{7} \sum_{i=0}^{m-1} \sum_{j=0}^{n-1} \frac{1}{7+4 \sin ^{2}(i \pi / m)+4 \sin ^{2}(j \pi / n)} \\
& +\frac{3}{14} n\left(m^{3}-m\right)+\frac{3}{14} m\left(n^{3}-n\right)+\frac{3 m^{2} n^{2}}{10}+\frac{m n}{2}, \\
& (i, j) \neq(0,0) .
\end{aligned}
$$

Proof. Supposing that the Laplacian eigenvalues of $L_{\left(C_{m} \square C_{n}\right)}$ are $\mu_{i j}$, one can readily see that

$$
\begin{gathered}
\mu_{i j}=4 \sin ^{2} \frac{i \pi}{m}+4 \sin ^{2} \frac{j \pi}{n}, \\
i=0,1, \ldots, m-1 ; \quad j=0,1, \ldots, n-1 .
\end{gathered}
$$

Applying Lemma 6, the following result is straightforward:

$$
\begin{aligned}
\mathrm{Kf}\left(T\left(T_{m \times n}\right)\right)= & \frac{18}{7} \mathrm{Kf}\left(T_{m \times n}\right)+\frac{3 m^{2} n^{2}}{10}+\frac{m n}{2} \\
& +\frac{24 m n}{7} \sum_{(i, j) \neq(0,0)} \frac{1}{7+\mu_{i j}} .
\end{aligned}
$$

Notice that $L_{\left(C_{m} \square C_{n}\right)}$ have $m n-1$ nonzero Laplacian eigenvalues, and

$$
\begin{array}{r}
\sum_{(i, j) \neq(0,0)} \frac{1}{7+\mu_{i j}}=\sum_{i=0}^{m-1} \sum_{j=0}^{n-1} \frac{1}{7+4 \sin ^{2}(i \pi / m)+4 \sin ^{2}(j \pi / n)}, \\
(i, j) \neq(0,0),
\end{array}
$$

where $i=0,1, \ldots, m-1$ and $j=0,1, \ldots, n-1$.

Consequently, the relationships between $T_{m \times n}$ and its variant networks $T\left(T_{m \times n}\right)$ for Kirchhoff index are as follows:

$$
\begin{aligned}
\mathrm{Kf} & \left(T\left(T_{m \times n}\right)\right) \\
= & \frac{18}{7} \mathrm{Kf}\left(T_{m \times n}\right) \\
& +\frac{24 m n}{7} \sum_{i=0}^{m-1} \sum_{j=0}^{n-1} \frac{1}{7+4 \sin ^{2}(i \pi / m)+4 \sin ^{2}(j \pi / n)} \\
& +\frac{3 m^{2} n^{2}}{10}+\frac{m n}{2}, \quad(i, j) \neq(0,0) .
\end{aligned}
$$

According to the results of Lemma 7, we can verify the formula for the Kirchhoff index of the total graph of $\mathrm{Kf}\left(T\left(T_{m \times n}\right)\right)$ from (24). Consider

$$
\begin{aligned}
\text { Kf } & \left(T\left(T_{m \times n}\right)\right) \\
= & \frac{18}{7} m n \sum_{i=1}^{m-1} \sum_{j=1}^{n-1} \frac{1}{4 \sin ^{2}(i \pi / m)+4 \sin ^{2}(j \pi / n)} \\
& +\frac{24 m n}{7} \sum_{i=0}^{m-1} \sum_{j=0}^{n-1} \frac{1}{7+4 \sin ^{2}(i \pi / m)+4 \sin ^{2}(j \pi / n)} \\
& +\frac{3}{14} n\left(m^{3}-m\right)+\frac{3}{14} m\left(n^{3}-n\right) \\
& +\frac{3 m^{2} n^{2}}{10}+\frac{m n}{2}, \quad(i, j) \neq(0,0) .
\end{aligned}
$$

This completes the proof of Theorem 11.

3.4. The Kirchhoff Index of $C\left(T_{m \times n}\right)$. We will explore the formula for estimating the Kirchhoff index in the clique-inserted graph of $T_{m \times n}$, denoted by $\mathrm{Kf}\left(C\left(T_{m \times n}\right)\right)$. 
Theorem 12. Let $C\left(T_{m \times n}\right)$ be clique-inserted graphs of $T_{m \times n}$ with any positive integer $m, n \geq 3$; then

$$
\begin{aligned}
K f( & \left.C\left(T_{m \times n}\right)\right) \\
= & 4 m n \sum_{i=0}^{m-1} \sum_{j=0}^{n-1} \frac{1}{3-\sqrt{5+2 \cos (2 \pi i / m)+2 \cos (2 \pi j / n)}} \\
& +4 m n \sum_{i=0}^{m-1} \sum_{j=0}^{n-1} \frac{1}{3+\sqrt{5+2 \cos (2 \pi i / m)+2 \cos (2 \pi j / n)}} \\
& +\frac{5}{3} m^{2} n^{2},
\end{aligned}
$$

where the first summation $i=0,1, \ldots, m-1 ; j=0,1, \ldots, n-1$, and $(i, j) \neq(0,0)$.

Proof. Noting that $S\left(T_{m \times n}\right)$ is $(r, 2)$-semiregular graphs and supposing that $S\left(T_{m \times n}\right)$ has $p$ vertices and $q$ edges, then obviously $p=3 m n, q=4 m n$, and $r=4$, respectively.

By virtue of Lemma 3,

$$
P_{L(G)}(x)=(-1)^{n}(x-(r+s))^{m-n} P_{G}(r+s-x) .
$$

Let $G$ be the graph $S\left(T_{m \times n}\right)$; that is,

$$
P_{L\left(S\left(T_{m \times n}\right)\right)}(x)=(-1)^{p}(x-(r+s))^{q-p} P_{S\left(T_{m \times n}\right)}(r+s-x) .
$$

From the definition of clique-inserted graph, one can immediately obtain that

$$
\begin{aligned}
P_{C\left(T_{m \times n}\right)}(x) & =(-1)^{p}(x-(r+2))^{q-p} P_{S\left(T_{m \times n}\right)}(6-x) \\
& =(-1)^{3 m n}(x-6)^{m n} P_{S\left(T_{m \times n}\right)}(6-x) .
\end{aligned}
$$

Obviously, it follows from Lemma 2,

$$
P_{S(G)}(x)=(-1)^{m}(2-x)^{m-n} P_{G}(x(r+2-x)) .
$$

Replace $x$ with $6-x$ in (30); moreover, $T_{m \times n}$ have $m n$ vertices and $2 m n$ edges; we have that

$$
P_{S\left(T_{m \times n}\right)}(6-x)=(-1)^{m n}(x-4)^{m n} P_{T_{m \times n}}(x(6-x)) .
$$

Based on (29) and (31),

$$
P_{C\left(T_{m \times n}\right)}(x)=(-1)^{4 m n}((x-4)(x-6))^{m n} P_{T_{m \times n}}(x(6-x)) .
$$

Since the roots of $x(6-x)=\mu_{i j}$ are

$$
3 \pm \sqrt{9-\mu_{i j}}
$$

where $\mu_{i j}$ are the Laplacian eigenvalues of $T_{m \times n}$ and $\mu_{i j}=$ $4 \sin ^{2}(i \pi / m)+4 \sin ^{2}(j \pi / n), i=0,1, \ldots, m-1 ; j=0,1, \ldots$, $n-1$.
It follows from (32) that the Laplacian spectrum of $C\left(T_{m \times n}\right)$ is

$$
\begin{aligned}
& \operatorname{Spec}_{L}\left(C\left(T_{m \times n}\right)\right) \\
& \quad=\left(\begin{array}{cccc}
4 & 6 & \mu_{i j}\left(C\left(T_{m \times n}\right)\right) & \mu_{i j}^{\prime}\left(C\left(T_{m \times n}\right)\right) \\
m n & m n & 1 & 1
\end{array}\right),
\end{aligned}
$$

where $\mu_{i j}\left(C\left(T_{m \times n}\right)\right)=3-\sqrt{9-\mu_{i j}}, \mu_{i j}^{\prime}\left(C\left(T_{m \times n}\right)\right)=3+$ $\sqrt{9-\mu_{i j}}, i=0,1, \ldots, m-1$, and $j=0,1, \ldots, n-1$.

Employing Lemma 1, (33), and the Laplacian spectrum of $C\left(T_{m \times n}\right)$, the following result is straightforward:

$$
\begin{aligned}
& \operatorname{Kf}\left(C\left(T_{m \times n}\right)\right) \\
& =4 m n\left(\frac{m n}{4}+\frac{m n}{6}+\sum_{(i, j) \neq(0,0)} \frac{1}{\mu_{i j}(C(G))}+\sum \frac{1}{\mu_{i j}^{\prime}(C(G))}\right) \\
& =\frac{5}{3} m^{2} n^{2}+4 m n\left(\sum_{(i, j) \neq(0,0)} \frac{1}{3-\sqrt{9-\mu_{i j}}}\right. \\
& \left.+\sum \frac{1}{3+\sqrt{9-\mu_{i j}}}\right) \\
& =4 m n \sum_{i=0}^{m-1} \sum_{j=0}^{n-1} \frac{1}{3-\sqrt{5+2 \cos (2 \pi i / m)+2 \cos (2 \pi j / n)}}, \\
& (i, j) \neq(0,0), \\
& +4 m n \sum_{i=0}^{m-1} \sum_{j=0}^{n-1} \frac{1}{3+\sqrt{5+2 \cos (2 \pi i / m)+2 \cos (2 \pi j / n)}} \\
& +\frac{5}{3} m^{2} n^{2} \text {. }
\end{aligned}
$$

Hence Theorem 12 holds.

Remark 13. The consequences of Lemma 7 and Theorems 9-12 above present closed-form formulae for immediately obtaining its Kirchhoff indexes in terms of finite various networks; however, the quantities are rather difficult to calculate directly.

\section{The Asymptotic Behavior of Related Kirchhoff Index}

We explore the asymptotic behavior of Kirchhoff index for the investigated networks above as $m, n$ tend to infinity. It is interesting and surprising that the quantity tends to a constant even though $\mathrm{Kf}(G) \rightarrow \infty$, as $m, n$ tend to infinity; that is,

$$
\lim _{m \rightarrow \infty} \lim _{n \rightarrow \infty} \frac{\operatorname{Kf}(G)}{m^{2} n^{2}}=C, \quad m, n \longrightarrow \infty
$$


Moreover, one can employ the applications of analysis approach to obtain the explicit approximate values of Kirchhoff index for the related networks.

Theorem 14. Let $L\left(T_{m \times n}\right)$ be line graphs of $T_{m \times n}$ with any positive integer $m, n$; then

$$
\lim _{m \rightarrow \infty} \lim _{n \rightarrow \infty} \frac{K f\left(L\left(T_{m \times n}\right)\right)}{m^{2} n^{2}} \approx 4.060 .
$$

Proof. According to (15) and the result of Lemma 8, we can derive that

$$
\begin{aligned}
\operatorname{Kf}\left(L\left(T_{m \times n}\right)\right) & =2 \operatorname{Kf}\left(T_{m \times n}\right)+\frac{m^{2} n^{2}}{4} \\
& =4.060 m^{2} n^{2} .
\end{aligned}
$$

Consequently,

$$
\lim _{m \rightarrow \infty} \lim _{n \rightarrow \infty} \frac{\operatorname{Kf}\left(L\left(T_{m \times n}\right)\right)}{m^{2} n^{2}} \approx 4.060 .
$$

The result is equivalent to $L\left(T_{m \times n}\right)$ having asymptotic Kirchhoff index,

$$
\operatorname{Kf}\left(L\left(T_{m \times n}\right)\right) \approx 4.060 m^{2} n^{2}, \quad m, n \longrightarrow \infty .
$$

Theorem 15. Let $S\left(T_{m \times n}\right)$ be subdivision graph of $T_{m \times n}$ with any positive integer $m, n$; then

$$
\lim _{m \rightarrow \infty} \lim _{n \rightarrow \infty} \frac{K f\left(S\left(T_{m \times n}\right)\right)}{m^{2} n^{2}} \approx 35.790 .
$$

Proof. Similarly, according to (18), we can easily verify that

$$
\begin{aligned}
\operatorname{Kf}\left(S\left(T_{m \times n}\right)\right) & =18 \mathrm{Kf}\left(T_{m \times n}\right)+\frac{3 m^{2} n^{2}+m n}{2} \\
& =35.790 m^{2} n^{2} .
\end{aligned}
$$

Hence,

$$
\lim _{m \rightarrow \infty} \lim _{n \rightarrow \infty} \frac{\operatorname{Kf}\left(S\left(T_{m \times n}\right)\right)}{m^{2} n^{2}} \approx 35.790 .
$$

Theorem 16. Let $T\left(T_{m \times n}\right)$ be total graph of $T_{m \times n}$ with any positive integer $m, n \geq 3$; then

$$
\lim _{m \rightarrow \infty} \lim _{n \rightarrow \infty} \frac{K f\left(T\left(T_{m \times n}\right)\right)}{m^{2} n^{2}} \approx 5.521 .
$$

Proof. Consider the summation term $\sum_{i=0}^{m-1} \sum_{j=0}^{n-1}(1 /(7+$ $\left.\left.4 \sin ^{2}(i \pi / m)+4 \sin ^{2}(j \pi / n)\right)\right)$.
Since

$$
\begin{aligned}
& \lim _{m \rightarrow \infty} \lim _{n \rightarrow \infty} \frac{1}{m} \frac{1}{n} \sum_{i=0}^{m-1} \sum_{j=0}^{n-1} \frac{1}{7+4 \sin ^{2}(i \pi / m)+4 \sin ^{2}(j \pi / n)} \\
& =\lim _{m \rightarrow \infty} \lim _{n \rightarrow \infty} \frac{1}{m} \frac{1}{n} \sum_{i=0}^{m-1} \sum_{j=0}^{n-1} \frac{1}{11-2 \cos (2 \pi i / m)-2 \cos (2 \pi j / n)} \\
& =\frac{1}{4 \pi^{2}} \int_{0}^{2 \pi} \int_{0}^{2 \pi} \frac{d_{x} d_{y}}{11-2 \cos x-2 \cos y} \approx 0.094 .
\end{aligned}
$$

The value in last line via the mathematic software MATLAB, which can obtain the result above.

Combining with (22), we can obtain that

$$
\begin{aligned}
\mathrm{Kf} & \left(T\left(T_{m \times n}\right)\right) \\
= & \frac{18}{7} \mathrm{Kf}\left(T_{m \times n}\right) \\
& +\frac{24 m n}{7} \sum_{i=0}^{m-1} \sum_{j=0}^{n-1} \frac{1}{7+4 \sin ^{2}(i \pi / m)+4 \sin ^{2}(j \pi / n)} \\
& +\frac{3 m^{2} n^{2}}{10}+\frac{m n}{2}, \quad(i, j) \neq(0,0) \\
\approx & 5.521 m^{2} n^{2} .
\end{aligned}
$$

So

$$
\lim _{m \rightarrow \infty} \lim _{n \rightarrow \infty} \frac{\operatorname{Kf}\left(T\left(T_{m \times n}\right)\right)}{m^{2} n^{2}} \approx 5.521 .
$$

Theorem 17. Let $C\left(T_{m \times n}\right)$ be clique-inserted graph of $T_{m \times n}$ with any positive integer $m, n \geq 3$; then

$$
\lim _{m \rightarrow \infty} \lim _{n \rightarrow \infty} \frac{K f\left(C\left(T_{m \times n}\right)\right)}{m^{2} n^{2}} \approx 38.591 .
$$

Proof. From the proof of Theorem 12, we know that

$$
\begin{aligned}
\mathrm{Kf}( & \left.C\left(T_{m \times n}\right)\right) \\
= & 4 m n \sum_{i=0}^{m-1} \sum_{j=0}^{n-1} \frac{1}{3-\sqrt{5+2 \cos (2 \pi i / m)+2 \cos (2 \pi j / n)}} \\
& +4 m n \sum_{i=0}^{m-1} \sum_{j=0}^{n-1} \frac{1}{3+\sqrt{5+2 \cos (2 \pi i / m)+2 \cos (2 \pi j / n)}} \\
& +\frac{5}{3} m^{2} n^{2},
\end{aligned}
$$

where the first summation $i=0,1, \ldots, m-1 ; j=0,1, \ldots, n-$ 1 , and $(i, j) \neq(0,0)$. 
As $m, n$ tend to infinity, it follows from the first summation term:

$$
\begin{gathered}
\lim _{m \rightarrow \infty} \lim _{n \rightarrow \infty} \frac{1}{m} \frac{1}{n} \sum_{i=0}^{m-1} \sum_{j=03-\sqrt{5+2 \cos (2 \pi i / m)+2 \cos (2 \pi j / n)}}^{n-1} \\
=\frac{1}{4 \pi^{2}} \int_{0}^{2 \pi} \int_{0}^{2 \pi} \frac{d_{x} d_{y}}{3-\sqrt{5+2 \cos x+2 \cos y}} \approx 9.037 .
\end{gathered}
$$

Similarly, it holds from the second summation term when $m, n$ tend to infinity,

$$
\begin{gathered}
\lim _{m \rightarrow \infty} \lim _{n \rightarrow \infty} \frac{1}{m} \frac{1}{n} \sum_{i=0}^{m-1} \sum_{j=03+\sqrt{5+2 \cos (2 \pi i / m)+2 \cos (2 \pi j / n)}}^{n-1} \\
=\frac{1}{4 \pi^{2}} \int_{0}^{2 \pi} \int_{0}^{2 \pi} \frac{d_{x} d_{y}}{3+\sqrt{5+2 \cos x+2 \cos y}} \approx 0.195 .
\end{gathered}
$$

Combining with the consequences of Theorem 12 and (50) and (51), it follows that

$$
\lim _{m \rightarrow \infty} \lim _{n \rightarrow \infty} \frac{\operatorname{Kf}\left(C\left(T_{m \times n}\right)\right)}{m^{2} n^{2}} \approx 38.591 .
$$

Summing up, we complete the proof.

\section{Conclusions}

Resistance distance was introduced by Klein and Randic as a generalization of the classical distance. In this paper, we have deduced the relationships between the toroidal meshes network $T_{m \times n}$ and its variant networks in terms of the Kirchhoff index via spectral graph theory. The explicit formulae for calculating the Kirchhoff indexes of $L\left(T_{m \times n}\right), S\left(T_{m \times n}\right)$, $T\left(T_{m \times n}\right)$, and $C\left(T_{m \times n}\right)$ were proposed for any positive integer $m, n \geq 3$, respectively.

The asymptotic behavior of Kirchhoff indexes has been investigated with the applications of analysis approach, and the explicit approximate values are obtained by calculations for the related networks. The values of Kirchhoff indexes with respect to various networks can be immediately obtained via this approach; however, the quantities are rather difficult to calculate directly.

\section{Conflict of Interests}

The authors declare that there is no conflict of interests regarding the publication of this paper.

\section{Acknowledgments}

The work of Jia-Bao Liu is partly supported by the Natural Science Foundation of Anhui Province of China under Grant no. KJ2013B105. The work of Xiang-Feng Pan is partly supported by the National Science Foundation of China under Grant nos. 10901001, 11171097, and 11371028.

\section{References}

[1] J. M. Xu, Topological Strucure and Analysis of Interconnction Networks, Kluwer Academic, London, UK, 2001.

[2] T. Shirai, "The spectrum of infinite regular line graphs," Transactions of the American Mathematical Society, vol. 352, no. 1, pp. 115-132, 2000.

[3] F. Zhang, Y.-C. Chen, and Z. Chen, "Clique-inserted-graphs and spectral dynamics of clique-inserting," Journal of Mathematical Analysis and Applications, vol. 349, no. 1, pp. 211-225, 2009.

[4] D. J. Klein and M. Randić, "Resistance distance," Journal of Mathematical Chemistry, vol. 12, no. 1-4, pp. 81-95, 1993.

[5] E. Estrada and N. Hatano, "A vibrational approach to node centrality and vulnerability in complex networks," Physica A: Statistical Mechanics and Its Applications, vol. 389, no. 17, pp. 3648-3660, 2010.

[6] X. Y. Liu and W. G. Yan, "The triangular kagomé lattices revisited," Physica A: Statistical Mechanics and Its Applications, vol. 392, no. 22, pp. 5615-5621, 2013.

[7] B. Zhou and N. Trinajstić, "A note on Kirchhoff index," Chemical Physics Letters, vol. 455, no. 1-3, pp. 120-123, 2008.

[8] I. Gutman and B. Mohar, "The quasi-Wiener and the Kirchhoff indices coincide," Journal of Chemical Information and Computer Sciences, vol. 36, no. 5, pp. 982-985, 1996.

[9] E. Estrada and N. Hatano, "Topological atomic displacements, Kirchhoff and Wiener indices of molecules," Chemical Physics Letters, vol. 486, no. 4-6, pp. 166-170, 2010.

[10] A. D. Maden, A. S. Cevik, I. N. Cangul, and K. C. Das, "On the Kirchhoff matrix, a new Kirchhoff index and the Kirchhoff energy," Journal of Inequalities and Applications, vol. 2013, article 337, 2013.

[11] J. B. Liu, J. Cao, X.-F. Pan, and A. Elaiw, "The Kirchhoff index of hypercubes and related complex networks," Discrete Dynamics in Nature and Society, vol. 2013, Article ID 543189, 7 pages, 2013.

[12] J. Liu, X.-F. Pan, Y. Wang, and J. Cao, "The Kirchhoff index of folded hypercubes and some variant networks," Mathematical Problems in Engineering, vol. 2014, Article ID 380874, 9 pages, 2014.

[13] J. B. Liu, X. F. Pan, J. Cao, and F. F. Hu, "A note on some physical and chemical indices of clique-inserted lattices," Journal of Statistical Mechanics: Theory and Experiment. In press.

[14] W. J. Xiao and I. Gutman, "Resistance distance and Laplacian spectrum," Theoretical Chemistry Accounts, vol. 110, no. 4, pp. 284-289, 2003.

[15] H.-Y. Zhu, D. J. Klein, and I. Lukovits, "Extensions of the Wiener number," Journal of Chemical Information and Computer Sciences, vol. 36, no. 3, pp. 420-428, 1996.

[16] D. Babić, D. J. Klein, I. Lukovits, S. Nikolić, and N. Trinajstić, "Resistance-distance matrix: a computational algorithm and its applications," International Journal of Quantum Chemistry, vol. 90, no. 1, pp. 166-176, 2002.

[17] J. L. Palacios and J. M. Renom, "Bounds for the Kirchhoff index of regular graphs via the spectra of their random walks," International Journal of Quantum Chemistry, vol. 110, no. 9, pp. 1637-1641, 2010

[18] J. L. Palacios, "Closed-form formulas for Kirchhoff index," International Journal of Quantum Chemistry, vol. 81, no. 2, pp. 135-140, 2001.

[19] I. Lukovits, S. Nikolić, and N. Trinajstić, "Resistance distance in regular graphs," International Journal of Quantum Chemistry, vol. 71, no. 3, pp. 217-225, 1999. 
[20] H. P. Zhang, Y. J. Yang, and C. Li, "Kirchhoff index of composite graphs," Discrete Applied Mathematics, vol. 157, no. 13, pp. 29182927, 2009.

[21] C. Arauz, "The Kirchhoff indexes of some composite networks," Discrete Applied Mathematics, vol. 160, no. 10-11, pp. 1429-1440, 2012.

[22] M. Bianchi, A. Cornaro, J. L. Palacios, and A. Torriero, "Bounds for the Kirchhoff index via majorization techniques," Journal of Mathematical Chemistry, vol. 51, no. 2, pp. 569-587, 2013.

[23] X. Gao, Y. F. Luo, and W. W. Liu, "Kirchhoff index in line, subdivision and total graphs of a regular graph," Discrete Applied Mathematics, vol. 160, no. 4-5, pp. 560-565, 2012.

[24] B. Mohar and Y. Alavi, “The Laplacian spectrum of graphs," in Graph Theory, Combinatorics, and Applications, vol. 2, pp. 871898, 1991.

[25] Z. You, L. You, and W. Hong, "Comment on 'Kirchhoff index in line, subdivision and total graphs of a regular graph," Discrete Applied Mathematics, vol. 161, no. 18, pp. 3100-3103, 2013.

[26] L. Ye, "On the Kirchhoff index of some toroidal lattices," Linear and Multilinear Algebra, vol. 59, no. 6, pp. 645-650, 2011. 


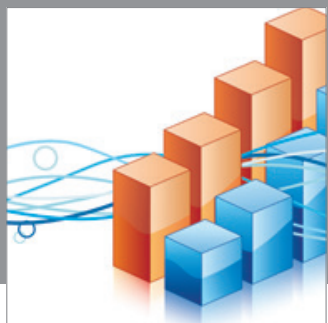

Advances in

Operations Research

mansans

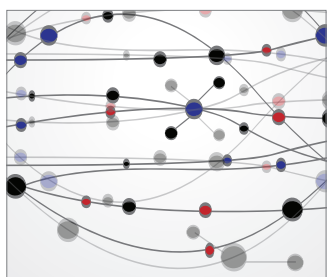

The Scientific World Journal
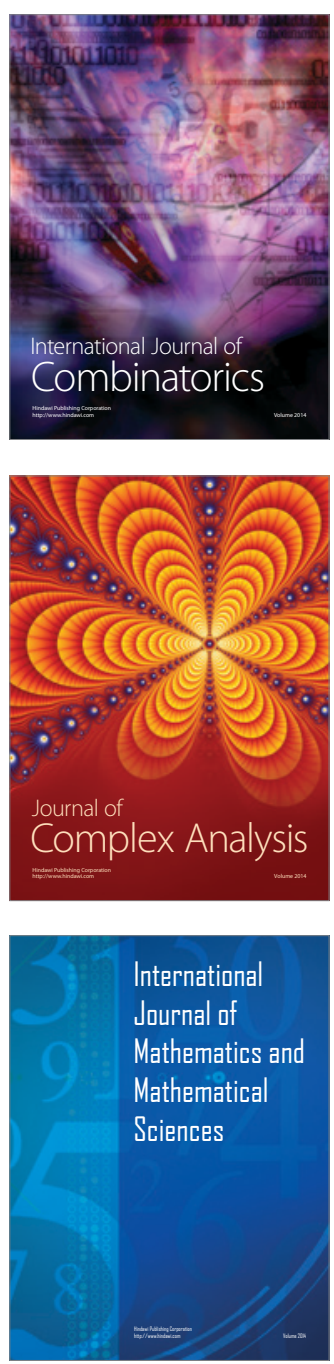
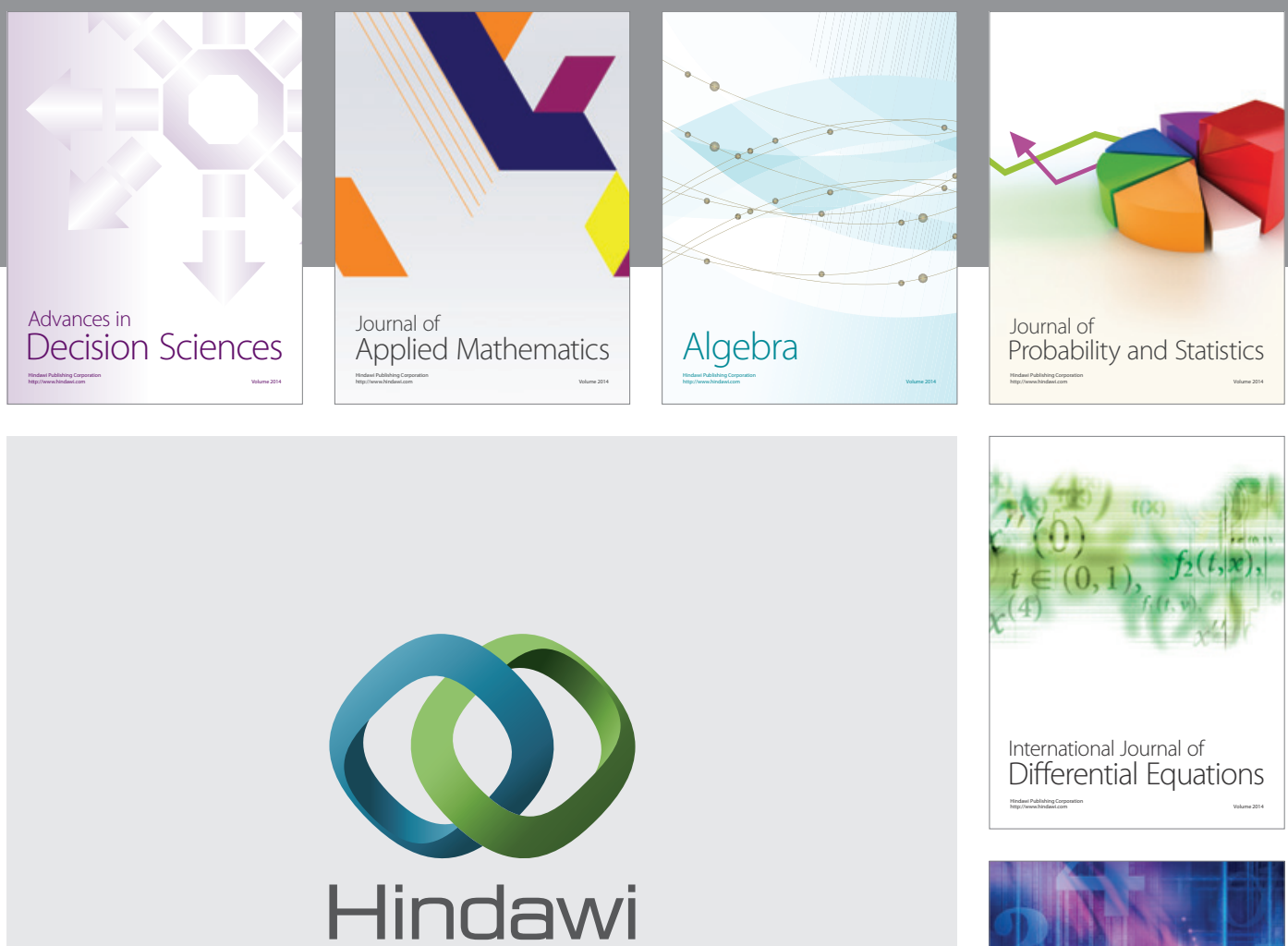

Submit your manuscripts at http://www.hindawi.com
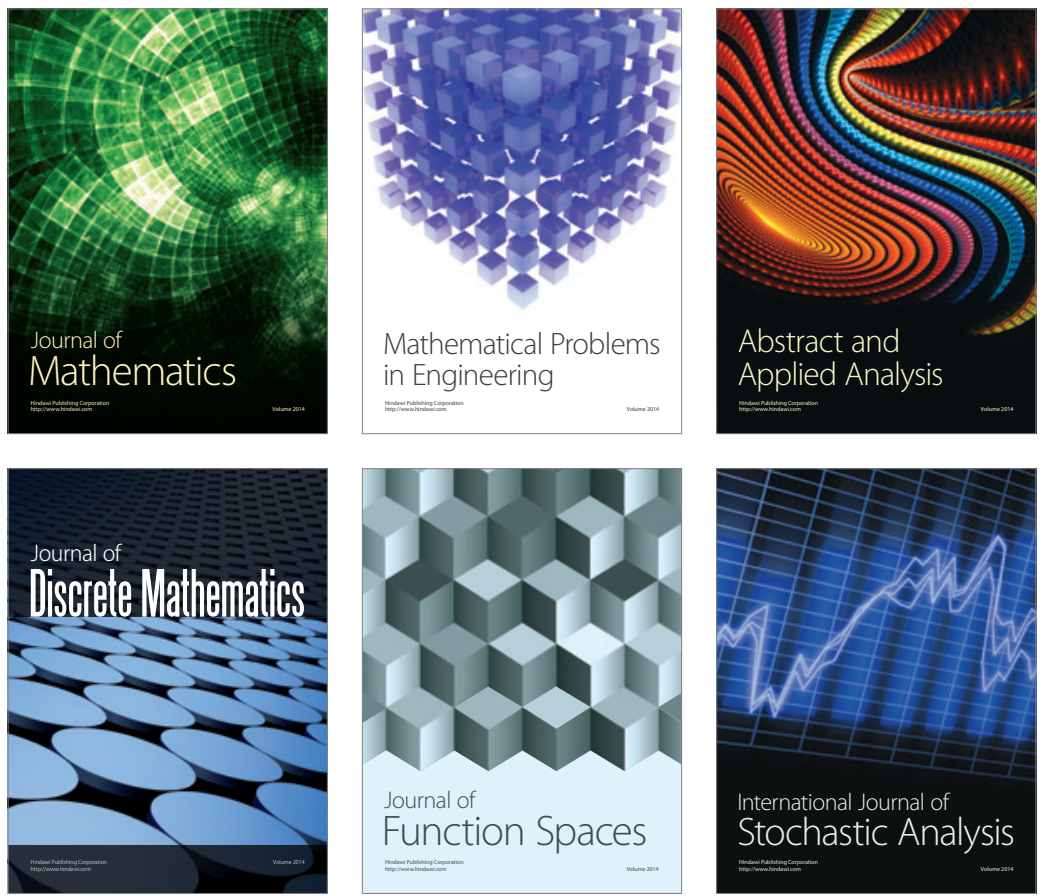

Journal of

Function Spaces

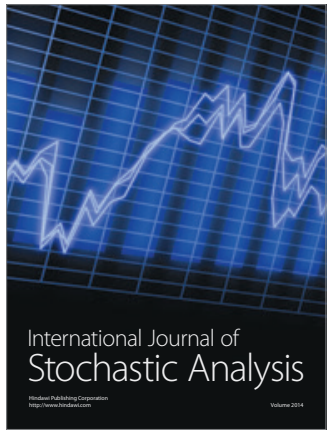

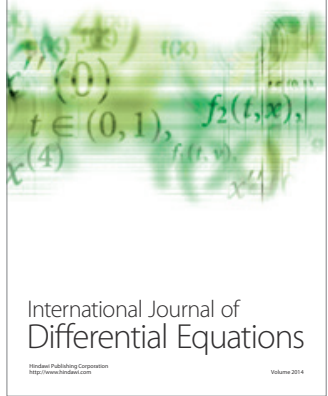
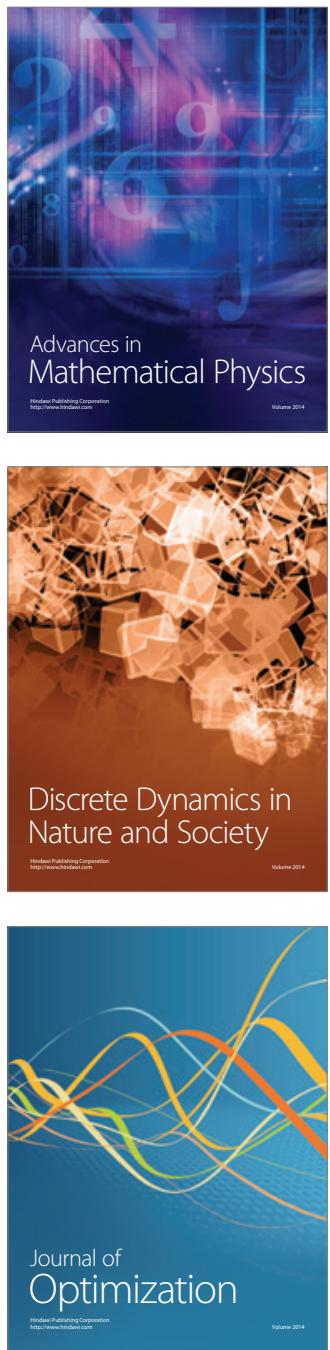